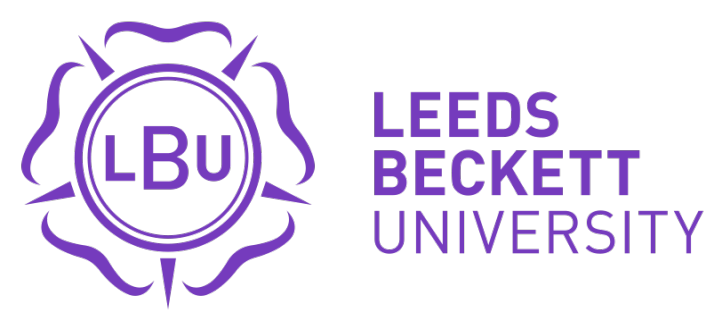

Citation:

Alsufyani, R and Chang, V (2016) Risk analysis of business intelligence in cloud computing. In: 2015 IEEE 7th International Conference on Cloud Computing Technology and Science (CloudCom). UNSPECIFIED, 558 - 563. ISBN 9781467395601 DOI: https://doi.org/10.1109/CloudCom.2015.84

Link to Leeds Beckett Repository record:

https://eprints.leedsbeckett.ac.uk/id/eprint/2692/

Document Version:

Book Section (Accepted Version)

The aim of the Leeds Beckett Repository is to provide open access to our research, as required by funder policies and permitted by publishers and copyright law.

The Leeds Beckett repository holds a wide range of publications, each of which has been checked for copyright and the relevant embargo period has been applied by the Research Services team.

We operate on a standard take-down policy. If you are the author or publisher of an output and you would like it removed from the repository, please contact us and we will investigate on a case-by-case basis.

Each thesis in the repository has been cleared where necessary by the author for third party copyright. If you would like a thesis to be removed from the repository or believe there is an issue with copyright, please contact us on openaccess@leedsbeckett.ac.uk and we will investigate on a case-by-case basis. 


\section{RISK ANALYSIS OF BUSINESS INTELLIGENCE IN CLOUD COMPUTING}

\author{
Raed Alsufyani \\ School of Computing, Creative Technologies, and \\ Engineering, Leeds Beckett University \\ Headingley Campus, Leeds LS6 3QS UK \\ e-mail: r.alsufyani5478@students.leedsbeckett.ac.uk
}

\author{
Victor Chang \\ School of Computing, Creative Technologies, and \\ Engineering, Leeds Beckett University \\ Headingley Campus, Leeds LS6 3QS, UK AND \\ e-mail: V.I.Chang@leedsbeckett.ac.uk
}

\begin{abstract}
The paper discusses the issues of risk analysis of Business Intelligence on the basis of Cloud platforms. The study gives an account on various aspects of the issue such as benefits and risks, financial appliance, and a factual process of data analysis. The paper attempts to address the issue in terms of empirical knowledge as long as numerous organizations face difficulties concerning appropriate application of Business Intelligence in the Cloud environment for purposes of risk forecasting and assessment.
\end{abstract}

Keywords- Business Intelligence, Cloud Computing, risk management, risk assessment, information technologies

\section{INTRODUCTION}

It is hard to deny the fact that cloud computing has made a revolution in the sphere of information technologies and opened a wide range of opportunities for this business segment [3]. Therefore, it is commonly applied to big enterprises while medium-sized companies are striving for better optimization of this technology [3]. A mong numerous functions of cloud computing, risk analysis has become a central issue for many organizations. The majority of firms usually utilize business intelligence for calculating and forecasting probability of risk and success, so that cloudbased technologies provide an obvious advantage [15]. It is certainly true to a particular extent, but there is a wide variety of implications, which have continued to arise. Thus, the following study focuses on the discussion and description of risks analysis in terms of business intelligence in the cloud environment. Having outlined the thesis and key terms of the paper, it is necessary to proceed to the next section.

\section{BUSINESS INTELLIGENCE AND CLOUD COMPUTING}

To start with, it is appropriate to give a brief account of business intelligence. It is becoming increasingly apparent that this technology is widely used in terms of business process management, especially in customer-driven firms. The term of Business Intelligence is commonly defined as a complex of integrated approaches for supporting IT-based managerial performance [3]. A distinct advantage of this technology relates to its ability to integrate with any business models. A particular advantage of business intelligence is based on providing data as a qualitative service [1]. In other words, customers are enabled to perform various tasks with the most optimum efficiency because of computationally reasoned data analysis, even though it is hardly integrated into external applications. With regard to cloud computing, it should be noted that it is considerably easier, faster, and more flexible information technology, which provides not only substantial computing capacity but also a global access approach. Therefore, scalability of data and its custom-regulated enhancement give the business segment a wide range of opportunities [15]. It can be forecasting, assessing, pricing, accounting, communicative, and tracking models. In this context, business are being reconsidered, emphasizing on the flexibility and strong sensitivity to environment and the use of strategic systems in real time. such as business intelligence and cloud computing.

\section{BENEFITS AND RISKS}

With regards to business intelligence in cloud computing environment, it should be admitted that cloud computing offers huge opportunities to the BI filed. Despite the development of cloud computing technology is currently at its infancy, with many issues still to be addressed. That is why some extents of difficulties can be potentially spotted, even though both technologies are commonly regarded as sophisticated ones. It can be explained by the fact that both technologies are facing a high demand nowadays, especially their integrated version. Thus, the lack of hardware or software capacity occurs only as a result of a poor budget of the related project. Cloud computing is revolting the economics of BI by making accessible the hardware, networking, security and software needed to create data marts and data warehouses on demand with a pay-as-usergo approach. On the contrary, cloud business intelligence presents a distinct risk to the performance of the enterprise. In fact, it is quite vulnerable to external environ ment, which cannot be initially considered in spite of the technology's ability to process substantial sets of data [5]. Therefore, it usually requires a wide time frame for its appropriate optimization. As a consequence, such integration does not fit every single company in the segment of medium and small business [4]. This type of business is oriented at other strategic objectives, so that sophisticated technologies will thwart them from a proper goal scoping.

Besides, quite dubious effects are produced from the perspective of source risks. It is becoming incredibly 
apparent that nearly a half of all SaaS-based applications does not render the same effectiveness in cloud environment. At the same time, the rest of the applications become highly-efficient as long as they deliver customer data with a minimum of obstacles and maximum of customer-driven intelligence, which is an evident trend from the perspective of the contemporary business information technologies. Hence, there has been a little agreement on risks and benefits of cloud business intelligence in regard to this aspect. Speaking about any risks in terms of legislation, it should be admitted that extremely scant evidence about any law violations are reported about the use of this technology [5]. One may argue, however, that it is quite a convenient tool for a wide range of fraud strategies. It is certainly true to some extent, but this statement can be easily refuted by the fact that the use of certain technology for malicious purposes is a matter of personal choice rather than technical and goal-setting essence of cloud business intelligence.

In contrast, it is necessary to mention that this technology presupposes less privacy as cloud-based technologies follow a principle "everyone accesses everyone". In such a way, any hierarchical barriers are eliminated, and the network obtains substantial flexibility. It is one of the primary purposes of cloud computing to be regarded as a benefit rather than a disadvantage [3]. However, it is pivotal to note that cloud computing has a large storage capacity as its flexibility is much safer than traditional data warehousing. As a result, risk and incident management are far simpler in cloud-based environment. The preceding statement can be referred to obvious security advantages as well. Cloud-based technologies require several layers of protection and always include reserve cloud storage to make data devastation a less dangerous threat to numerous firms and organizations [13]. Still, effective protection requires nearly the same costs as well as the initial cloud platform, as a result, budgeting is expected to considerits top break-even margins.

Eventually, it is worth admitting that costs are usually needed for staff training since human resources and newly outlined information technology have to be clearly acknowledged. Therefore, this aspect cannot belong to the benefits of business intelligence in cloud environment. It makes many organizations refuse utilize it even though most of the benefits and risk reductions are explicit for them [13]. However, every single company should decide whether it is necessary to incorporate Cloud Business Intelligence in its managerial performance in accordance with estimation of the following source of BI benefits.

\begin{tabular}{|l|c|}
\hline Benefits & Rank \\
\hline Improve satisfaction of work & 1 \\
\hline
\end{tabular}

\begin{tabular}{|c|c|}
\hline Opportunity to develop new skills & 1 \\
\hline $\begin{array}{c}\text { Opportunity for organis ational } \\
\text { growth }\end{array}$ & 1 \\
\hline Improved status & 2 \\
\hline Removal of tedious work & 2 \\
\hline $\begin{array}{c}\text { Opportunity to manage inco me and } \\
\text { outgoings }\end{array}$ & 3 \\
\hline
\end{tabular}

Table 1. Source of BI benefits

[16]. In the same way, potential risks of adopting Cloud Business Intelligence should be assessed:

\begin{tabular}{|c|c|}
\hline Risks & Rank \\
\hline Lack of supporting resources & 1 \\
\hline Lack of understanding of the cloud & 1 \\
\hline Departmental downsizing & 2 \\
\hline Uncertainty with new technology & 2 \\
\hline $\begin{array}{c}\text { Increased dependence on external third } \\
\text { party }\end{array}$ & 3 \\
\hline Decrease of satisfying work & 3 \\
\hline
\end{tabular}

Table 2. Potential risks of adopting Cloud BI

[16]. It is becoming evidently explicit that the study focuses particularly on risks during the adoption. For instance, the most widespread adoption risk is related to choice of the most appropriate pricing model [14]. Actually, one of the following consulting project will be dealing with this issue in terms of data analysis.The metrics for measuring risks are understanding the Cloud-based technologies themselves, certaint of appliance the new technology, and increase of satisfying work. The mentioned risk is quite widespread as it causes many companies to adopt risky initiatives in order to enhance its strategic positioning.

\section{FINANCIAL MODEL}

In regards to the risks described in the previous section, it is worth saying that they can be still classified even though their probability is quite vague. Traditional Organisational Sustainability Modelling suggests that risks can be allocated to such metrics as Technical, Financial (Costs), and User. Since these metrics presuppose different corporate focuses, the results should be delivered independently while the input data requires consideration of external factors.

It is becoming increasingly apparent that the main is sue is based on the adoption of risks. In other words, any business initiatives are followed by certain risks always so that the central dilemma relates to the question whether a particular initiative is worth implementation. Hence, firms 
try to find various solutions to risk adoption so that Business Intelligence on a Cloud-based platform has become a certain trend.

Needless to say, every single firm attempts to develop a custom risk analysis model. Nevertheless, the general principle of risk assessment via business intelligence in cloud is the following: the majority of companies utilize OSM model, which consists of two independent elements of probability computing and statistical data regression. However, these components have a common output throughout $3 \mathrm{D}$ visualization and mathematica applications. It can be any other output interpretation, but visualized data renders a maximum of intelligence, which is pivotal to business decision-making. These processes are usually accompanied by quality assurance tools, which trace, correct, and report about existing or potential errors in calculations [7]. As a result, a standard hybrid computing model works in a way that is presented in the picture below.

It is quite obvious that such a model provides multi-sided accessibility, which is important to an enterprise's departments.Since the main problem is lack of data on a stage of risk adoption, a high accuracy of forecasts is essentially needed. That is why the most applicable model is chosen manually by picking up a model, which 'fits' the accuracy of calculation not less than $95 \%$ of probability.

\section{DATA ANALYSIS}

To start the analysis, it is necessary to mentioned that both sources of data are anonymous. The first data set operates with calculations of risks regarding return on investments in the company in relation to its impacts on the future stock market value. The data are stored in the cloud, and the computing is conducted via a standardized linear function, which is the following: $a=r f+(\beta \times(e-r f))$, where $\mathrm{a}$ is actual return on investments, $\mathrm{rf}$ - is a free risk rate, $\mathrm{e}-$ is expected return, and beta is a value of representation uncontrolled risk rate and measure. There is a wide range of simulation probabilities throughout this function, but this model utilizes a beta regression. That makes calculations maximally accurate since hidden segments are included even though they do not obtain a distinct value in the function [6]. What is more, key statistical data will be explicit so that the company's personnel do not have to pay attention to various statistical details. Still, a function of $p$-value should be applied. This function determines the initial probability of deviation. Normal forecast deviation varies from $1 \%$ to $5 \%$. Since positive and negative deviations are needed the function of $\mathrm{p}$-value can look like the following: $\mathrm{P}(\mathrm{t})=2 \mathrm{~min}$ $\left(\mathrm{P}_{0}, \mathrm{P}\right)$, where $\mathrm{P}(\mathrm{t})$ is the value of probability, $\mathrm{P}_{0}$ is a null hypothesis, and $\mathrm{P}$ - hypothetical deviation [10].P-value is a function that is used for calculation the preliminary deviation at taking risk. That is why null hypothesis is also considered as standard one can be indicated inappropriately at this stage of forecast testing.
The consulting project deals with risk management as a service in terms of computing appropriate pricing rates and related risks in relation to adequacy of prices towards a new target segment of a company. Again, this consulting project is supposed to ensure that none of the hidden nodes can be left beyond the terms of calculations. In fact, this data set is computed via $p$-value function. The results are expecyed tp witness about the deviation of forecast not to exceed $1 \%$. That implies the fact that a null hypothes is $\left(\mathrm{P}_{0}\right)$ should very close to a standard deviation $(\mathrm{P})$ ititially. As long as deviation is calculated regarding positive and negative dimensions, the related test results are calulctaed in both directions accordingly (2min) [10]. Hence, the firm identified its pricing strategy quite accurately.

Consequently, the results for the consulting projectare the following. First of all, beta occurred to determine the low break-even marg in of risk and standard error becomes explicit. This output result can be interpreted as an evidence that the company will not increase its market value in case the revenues will go on the one position below or above the break-even point. It is worth mentioning that standard error is not visible in terms of intentional calculations as long as such a type of errors usually belongs to experimental data. Still, this aspect has to be considered since it depicts potential scenarios of the risks and investment curve. Furthermore, automatic correlation was between -1 and 1 so that accuracy of error should be adjusted to $1 \%$. Hence, there is a one percent of probability that market value keep growing at the same rate of ROI. That is why such method of risk analysis does not presuppose any additional procedures for adjustment or alignment of output data. Hence, it is widely used in big enterprises, which operate with substantial finance and related data. Conversely, the consulting project is generally applicable to small and medium firms.

As the output results of the consulting project have been witnessed, this approach creates a reliable break-even bottom line in order to enable the company to be aware of any risky amendments in its investments and related financial operations. However, it does not presuppose external error recognition and correction. It provides sufficient but not output information as long as the decisionmaking process is still has to be conducted "manually", even though the model is capable of providing considerable amounts of business-related intelligence. Therefore, a quality assurance tool is usually applied in order to make output results as reliable as possible [5]. Needless to say, it makes cloud business intelligence excessively complicated, but it renders a maximum of reliability, especially in terms of risk analys is. This approach, however, has its distinct advantages in comparis on with the second set of data. Thus, it is es sential to discuss it as well.

To be more specific, the results for the variancegamma process determine a percentage of deviation from a required standard. In such a way, the related organization deviated on $0,7 \%$, which is not crucial for its financial performance. This function is useful for regulation and 
control of the current performance of the organization [8]. Moreover, it is still able to forecast the same deviation rates for the future periods. It requires standard input data and any aligning meanings, which can be integrated with this function. On the contrary, this approach evidently lacks an additional vector, which can obtain a value of external factors such as stock market fluctuations, political and sudden economic instability, social events, and etc. It should be developed as a separate set of functions in order to cross points of both vectors is an area of a distinct risk. Also, it leaves a decision-making process to be a human responsibility in spite of the fact that it provides error-free data. This sub-model shows a likelihood of a certain financial danger; consequently, business decision-making depends heavily on the external environment.

As for the least square method, it provides fast simulations, which can be easily converted in any other form of interpretation. Usually, these are percentage rates or simplified C \# linear functions. Still, this approach is not recommended on early stages of the company's performance because business intelligence will be not able to process a little data properly. In addition, it will become extremely redundant because of nearly empty cloud. Therefore, it is strongly advisable to conduct profound optimization of software and cloud environment. Otherwise, the organization is likely to face a drastic loss as such a type of information technologies implies substantial costs. Output results, however, is not the final stage of business intelligence in cloud environment [8].

The following and the last stage is data visualization. In regard to the consulting project, results are presented in a 3D form; three dimensions address three vectors of business intelligence computing of risk probabilities. The dimension $\mathrm{X}$ corresponds to the vector of potential risks, $\mathrm{Y}$ is related to the factual risks of the company, and the dimension $\mathrm{Z}$ depicts the risk-free zone of probabilities. Such a form of output data representation makes business-related calculations considerably easier as the personnel does not have to get into separate details of the firm's statistics. Moreover, output results can be available to external stakeholders such as investors, governmental regulatory organs, and even customers of the company. It is especially a significant contribution to the firm's financial performance since it sets the objective according to the forecasted returns on investment rates. Besides, the key aspects of the calculations are also depicted in the visualization.

Three dimensions are mutually related and rate the automatic correlation. As it has been mentioned before, the rate of automatic correlation was between -1 and 1 as it is accordingly reflected in the visualization. The major advantage of this form of visual representation of output data is a drastic reduction of complexity and time. That renders flexibility and scalability to the company's financial modelling. It is worth saying that this type of visualizing stage is recommended for application in medium and small business as long as it is capable of substantially contributing to projective and objective scoping. The result has to be interpreted as an evidence of a minor deivation from acceptable prciign strategy. In other words, the firm has to align its prcing on $0.7 \%$. Neeeldess to say, this deviation is not risky. Big enterprises are also recommended to utilize this form of data representation since they usually operate with big amounts of complicated data. Still, the second data set is represented in a simpler way, so it can be also advised for medium-sized firms too. Overall, it is to be noted that botth data sets relate to the adoption risks of decrease of satisfying work so that both companies were forced to take a drastic action that implied risks.

The second set of data is represented in $2 \mathrm{D}$, but it is essential to mention that data that follows Gamma distribution is depicted in a special way. Therefore, the most possible probabilities are highlighted. This is an obvious advantage and it will be explained further. To return to the subject of the visualization of the second set of data, it is worth mentioning that infrequent probabilities are not shown at all. One may argue that it is a drastic drawback, but it is appropriate to make a comment that every single company tries to scope its performance around a particular set of objectives, which are connected to according data and statistics. In such a way, a prediction can be made with $95 \%$ of accuracy. It can be explained by the fact that slope of regression line usually demonstrates a correlation between actual regression and a predicted one. Hence, the formula can be rewritten as follows $\beta=(\mathrm{e}-\mathrm{rc})$ : (a- rc). Generally speaking, such a way of data representation is more intelligent and simplified. It makes certain suggestions concerning business decision-making and eliminates unnecessary data to expect a minimum of potential inaccuracies. As a result, the following diagram demonstrates that this model is a good fit, but some uncertainty can be spotted at the regression line.

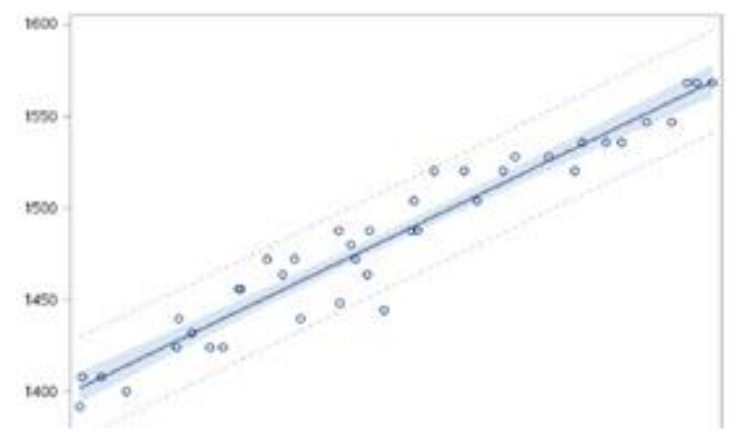

Fig. 1. Fit plot for tenbd

In fact, the diagram shows that the majority of output results comply with the regression line. Therefore, it means that the chosen model is $95 \%$ accurate since the majority of results stick to the regression line. It is pivotal to note that appliance of r-suqare is essential. That vakue actually 
determines how close the data are to the line of regression. Generally speaking, it should be equal to accuracy rate so that it should be $95 \%$ for the current data analys is.

In the larger context both data sets can be depicted as the following.
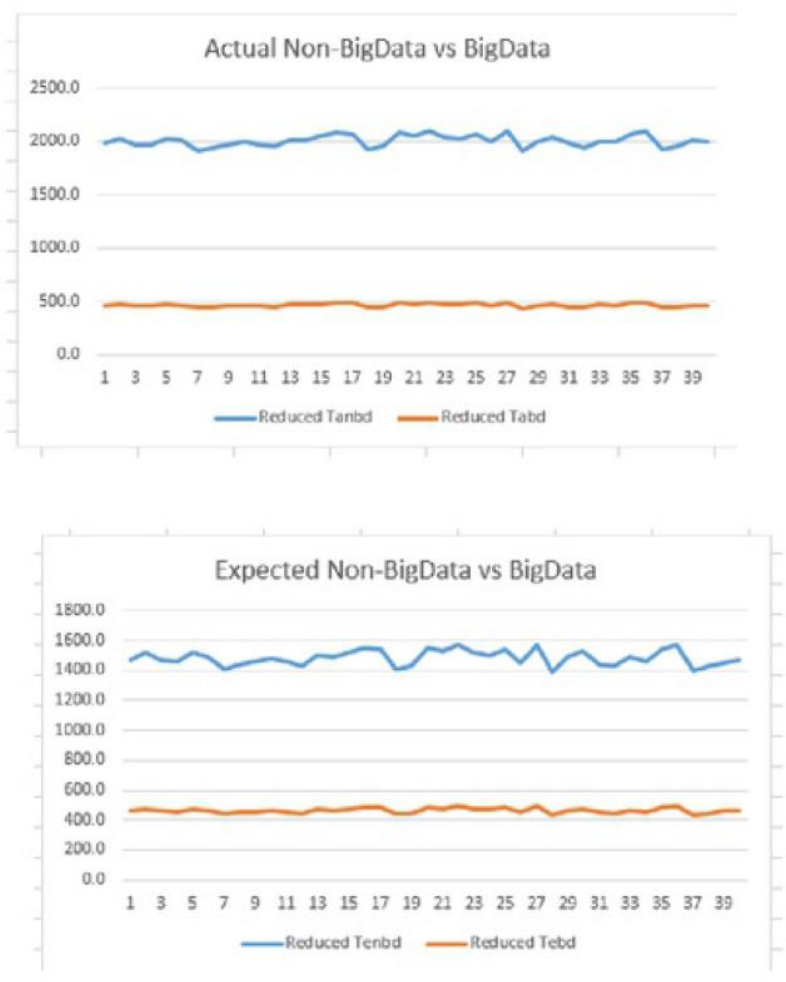

Fig. 2. Expected Non-BigData vs BigData

Needless to say, it is appropriate to give an account on the mean squared value that determines difference between estimated and actual results. Accordingly, the difference between two output results is expected to be no more than $5 \%$ as the model is attempting to guarantee $95 \%$ of accuracy.The last test of accuracy can be rendered via fmeasure function. This function demonstrates the relation between correct and positive results so that a combination with value 1 and more indicates a successful sampling. This function is hardly applicable to the business case, but some circumstances like implementation of innovative technologies ordrastic change can be tested by f-measure.

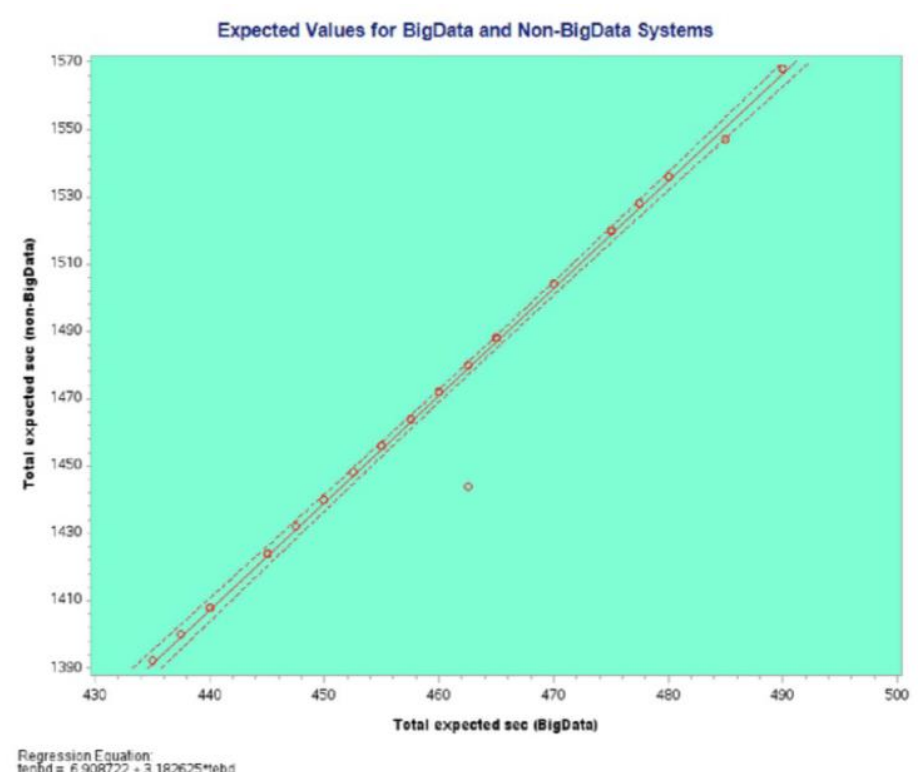

Fig. 3. Observed value and Estimted regression line

The diagrams depict the difference between results of operating BigData accuracy with Non-Big Data. Thus, appliance of both types of data can be aligned with the regression line in Cloud Business Intelligence. To the greatest extents, both regressions can be integrated throughout overall optimization of Cloud architecture. To return to the subject, it is worth saying that data in the consulting project undergo the accuracy tendency demonsstared in the illustration above.

It is becoming increasingly difficult to ignore the fact that this visualization is mainly applicable to small or medium companies. It can be explained by its ability to focus on current problems, without involving all data. Instead, it requires QaaS applications since no incorporated quality assurance tools are installed together with such layout of cloud business intelligence. Thus, it is supposed to be mainly business intelligence-driven rather than based on a cloud platform. As it has been mentioned previously, small-sized companies have to ensure an in-depth optimization of such technologies. This requirement is also underpinned by a substantial degree of business intelligence's integrative capacities. Eventually, it is necessary to admit that the analys is of two data sets has revealed specific peculiarities of risk analysis via cloud business intelligence in big as well as small-sized companies. Both types have their advantages and disadvantages, and it is reasonable to note that they can be combined in order to fill potential gaps and increase overall effectiveness. Since the financial models are suggested for integration, which is quite natural for Business Intelligence, it is reasonable to align the entire Cloud services with Business Intelligence as it is the main determinant of informational strategic perspective of the firm. That is why 
the following architecture is recommended as it provides meaningful scalability, security, and desired integrity:

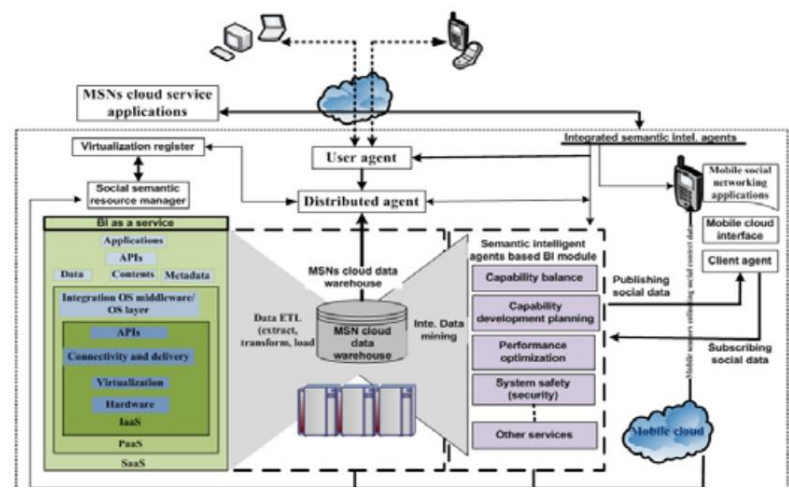

Fig. 4. Proposed ICDM platform of Cloud based BI [11]

\section{CONCLUSION}

All in all, it should be admitted that the study has given an account on risk analysis in terms of business intelligence in cloud environment. The paper has described the key purposes of both technologies. As a consequence, advantages and disadvantages of such integration have been discussed. The study has also investigated to the most commonly used financial model, which is consists of two linear functions. In order to conte xtualize these findings, the study has conducted the analysis of two data sets. As a result, peculiar features of computing, accuracy, visual representation, and applicability to business environment have been issued. Finally, it is worth admitting that cloud business intelligence is evidence of a distinct progress within the sphere of information technologies, even though much of improvements are expected in the nearest future.

\section{REFERENCES}

[1] H. Al-Aqrabi, L. Liu, R. Hill and N. Antonopoulos, 'Cloud BI: Fut ure of business intelligence in the Cloud', Journal of Computer and System Sciences, vol. 81, no. 1,pp. 85-96, 2015.

[2] H. Baars and H. Kemper, 'Integration von Big Data-Komponenten in die Business Intelligence', CON, vol. 27, no. 4-5, pp. 222-228, 2015.Campegemini 2011, Cloud computing in banking, Capegemini Analysis 2011, viewed 4 June 2015, $<$ http://www.uk.capgemini.com/resource-fileaccess/resource/pdf/Cloud_Computing_in_Banking.pdf $>$..

[3] D, Catteddu \& G ,Hogben, "Cloud computing: benefits, risks and recommendations for information security", European Network and Information Security Agency,2009

[4] V, Chang "A proposed model to analyse risk and return for a large computing system adoption", Physical science and engineering, doct oral thesis, 2013 viewed 5 June 2015, <http://eprints.soton.ac.uk/361523>.

[5] V., Chang, R. J.,Walters, \& G., Wills. "Review of Cloud Computing and existing Frameworks for Cloud adoption".(2014)

[6] M. Ramachandran and V. Chang, 'Financial Software as a Service:', International Journal of Organizational and Collective Intelligence, vol. 4, no. 3, pp. 65-89, 2014.

[7] V., Chang. "The business intelligence as a service in the cloud. Future Generation Computer Systems", 37, 512-534.(2014)..

[8] H. Chen, L. Li and Y. Sun, 'Risk Assessment of Aero Engine Failure based on Monte Carlo Simulation', Procedia Engineering, vol. 80, pp. 415-423, 2014.

[9] G. Dallal, The little handbook of statistical practice.: Gerard V. Dallal, 2012..

[10] G. B, Gebremeskel, An Architecture of Intelligent Cloud Data Mining Platform for Optimization of Cloud Based Business Intelligence', JCIT, vol. 8, no. 4, pp. 304-312, 2013.

[11] A., Khajeh-Hosseini, I., Sommerville, \& I., Sriram, "Research challenges for enterprise cloud computing". arXiv preprint arXiv:1001.3257.(2010).

[12] A. Khajeh-Hosseini, D. Greenwood, J. Smith and I. Sommerville, The Cloud Adoption Toolkit: supporting cloud adoption decisions in the enterprise', Softw. Pract. Exper., vol. 42, no. 4, pp. 447-465, 2011.

[13] L., Menon, \& B., Rehani. "Business intelligence on the cloud - over view and use case", 2014.

[14] M., Mircea, 'Strategy for selecting a business intelligence solution', Informatica Economica, vol. 1, no. 45, pp. 103-109, 2008

[15] R. Tunowski, 'Business Intelligence in Organization. Benefits, Risks and Developments', Przedsiebiorczosc i Zarzadzanie, vol. 16, no. 2, 2015. 